\title{
Study on Influence of Excitation System Parameters on Transient Characteristics of Power Units
}

\author{
Qiao Chen ${ }^{1}$, Jun He ${ }^{1}$, Wentao Huang ${ }^{1}$, Xin Shu ${ }^{1}$, Li Wan ${ }^{1}$ \\ State Grid Hubei Electric Power Research Institute, Hubei Wuhan 430077
}

Keywords: excitation control system, time constant, transient characteristics, stability of the power system, excitation control model.

\begin{abstract}
The engine excitation control system has significant effect on the static stability, dynamic stability and transient stability of the power system. In power system stability calculation, the model and parameters are used to correct the excitation system. Excitation system parameters are numerous, such as the excitation time constant, the static gain of the excitation time constant, the static gain of the excitation system, the PID time constant, the open circuit time constant of the generator excitation windings, etc. The influence of the above parameters on the transient characteristics of the generator set is studied in the case of a three machine system in Hubei province. The research results will provide reference for the optimal of the parameters of the excitation system model.
\end{abstract}

\section{Introduction}

Excitation control system of generators have a significant impact on the static stability, dynamic stability and transient stability of the power system。Using the right excitation system model and parameters in the power system stability calculation has critical influence on the calculation results. Therefore, the mathematical model and parameters that can properly reflect the actual operation condition of the equipment are needed to make the calculation results more reliable. The new stability guide requires more precise models for generator, and actual excitation system model and parameters for the calculation ${ }^{[1-4]}$. When the differences between the generator no-load $5 \%$ step response test results and simulation results are comparatively large, it is necessary to optimize the related parameters of excitation system. But there are many actual excitation system parameters that affect the results, such as exciter time constant, the total static excitation system gain, PID link time constant, generator field winding constant time with the stator open circuited, so it is difficult to find out which single parameter affects the output curves of the generator no-load 5\% step disturbance simulation. Therefore, it is urgent to study the impact of key excitation system parameters on generator transient characteristics to provide a basis for the identification, checking and optimization of generator excitation parameters.

Next, we take a three-machine excitation system of a generator in Hubei Province as an example to study the impact of key excitation system parameters on the generator transient characteristics.

\section{Generator Excitation System Model and 5\% Step Response Dynamic Characteristics}

In PSASP program, there are 14 kinds of excitation regulator models from type 1 to type 14, based on the type of exciter and rectifier whether controllable, with or without deputy exciter etc. Take type 3 as an example

We often use the generic models in PSASP program, but manufacturers use custom model in the actual field. A unit in Hubei Province with three-machine brush excitation system, using the 353MVA turbo generator of Harbin Electrical Machinery Plant, configured type PCS-9410 microcomputer excitation regulator of NARY Relaying Company. Its excitation system AVR transfer function block diagram is shown in figure 2 . 


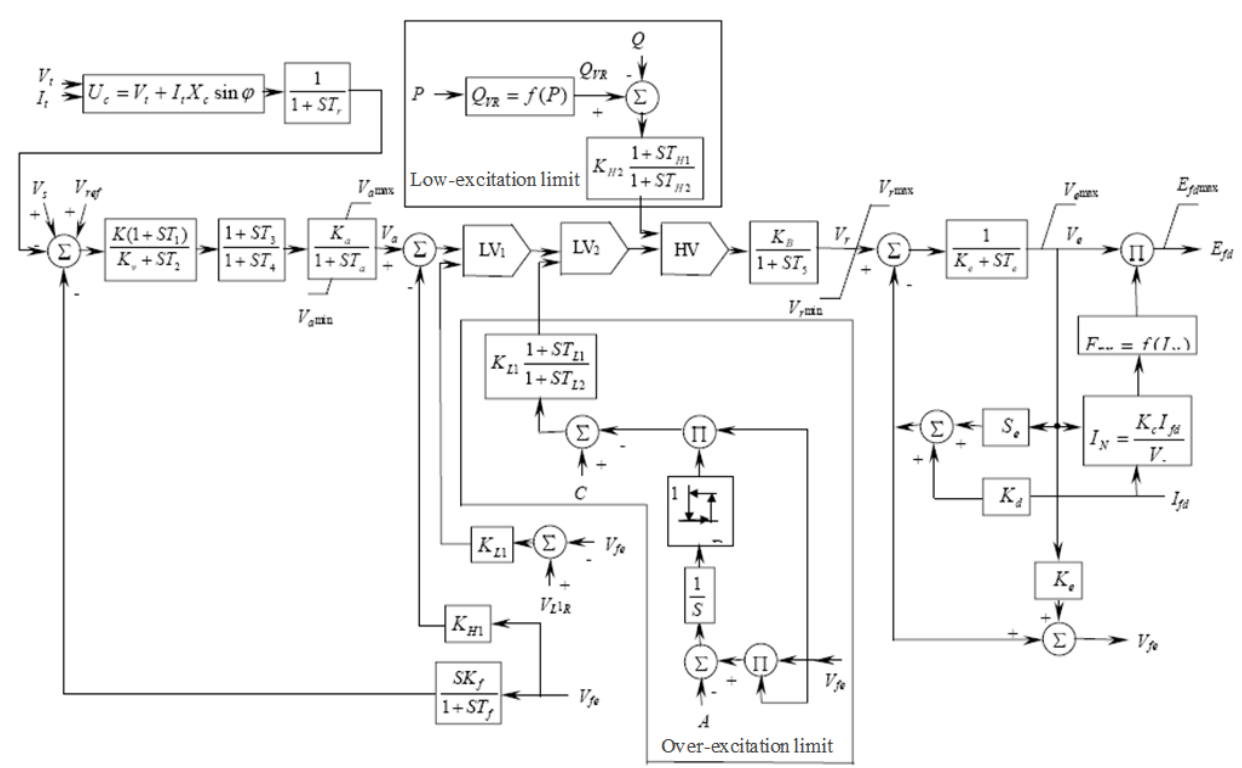

Fig.1 Excitation system block diagram of Type 3

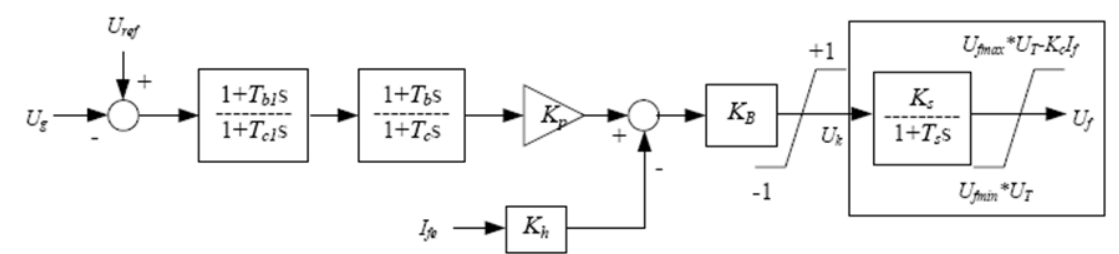
Note: $U_{\text {ref: }}$ Voltage reference value
$U_{g}$ : Voltage measurements
$K_{p}$ : Open loop gain coefficient
$T_{b}, T_{c}, T_{b 1}, T_{c 1}$ : Time constant
$K_{B}$ : Phase shift gain coefficient
$K_{h}$ : Hard feedback coefficient
$I_{f e}$ : Exciter field current

$$
\begin{aligned}
& U_{k}: \text { Triggering angle cosine } \\
& U_{f}: \text { Output voltage of Rectifier Bridge } \\
& K_{s}: \text { Top voltage coefficient } \\
& T_{s}: \text { Time lag of phase shift and rectifying } \\
& U_{T}: \text { Per unit voltage of Rectifier Bridge AC side } \\
& K_{e}: \text { Thyristor commutation voltage drop coefficient } \\
& I_{f}: \text { Generator field current }
\end{aligned}
$$

Fig.2 Excitation system AVR transfer function block diagram of a Unit in Hubei

Parameter test method is: Simulate and calculate a step response of established model and parameters of excitation system with the same step size as in the field test. Calculate the rise time tup (s), peak time tp(s), overshoot Mp (\%), setting time ts(s) and oscillation times $\mathrm{N}$ of the measured and simulated generator no-load voltage step response respectively, as it shown in figure 3.

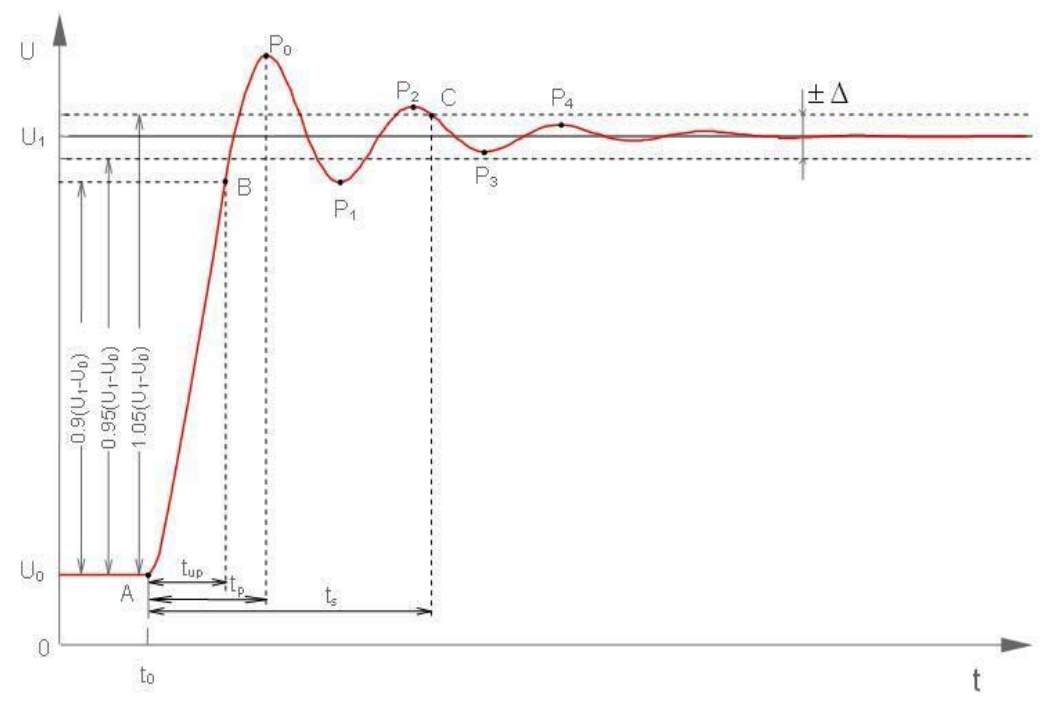

Fig3. Step indicator diagram

Wherein the rise time tup (s) refers to the time from the start time $t_{0}$ to the time when the 
variation of controlled variable first reach $90 \%$ of step size. Peak time tp (s) refers to the time from the start time $t_{0}$ to the time when controlled variable first reach peak value. Settling time ts (s) refers to the time from the start time $t_{0}$ to the time when the absolute difference value of controlled variable's variation and amount of step is less than $5 \%$. Overshoot $M p(\%)$ refers to the percentage of difference between the peak value and the final steady-state value of the controlled variable relative to the amount of step; Number of oscillations $\mathrm{N}$ refers to the oscillation times of controlled variable in the adjustment time.

\section{The Equivalent Processing of Generator Excitation Control Model}

Since statements of PSASP program and generator manufacturers about excitation system PID correction link are inconsistent, it is necessary to do equivalent processing to the transfer function in figure 1 and figure 2, obtaining new differential link lead-lag time constants, and new integral link lead-lag time constants. The design of manufacturer's parameters is shown in table 1.

Table.1 AVR parameters of a unit in Hubei

\begin{tabular}{|c|c|}
\hline Voltage regulation gain $\mathrm{Kp}$ & 20.0 p.u. \\
\hline Phase compensation time Tb & $2.00 \mathrm{~s}$ \\
\hline Phase compensation time Tc & $30.00 \mathrm{~s}$ \\
\hline Phase compensation time Tb1 & $0.30 \mathrm{~s}$ \\
\hline Phase compensation time Tc1 & $0.02 \mathrm{~s}$ \\
\hline
\end{tabular}

After above parameters converted to series PID transfer function parameters, the transfer function expression is:

$$
\Phi(s)=20 \frac{1+2 s}{1+30 s} \frac{1+0.3 s}{1+0.02 s}
$$

Modeling and simulation parameters is shown in table 2:

Table2. AVR parameters and their initial values

\begin{tabular}{|c|c|c|c|c|}
\hline No & Parameter & $\begin{array}{c}\text { Initial } \\
\text { Values }\end{array}$ & Unit & Note \\
\hline 1 & $\mathrm{~K}$ & 20 & & Gain \\
\hline 2 & $\mathrm{~T} 1$ & 0.3 & $\mathrm{~s}$ & $\begin{array}{c}\text { Differential link lead time } \\
\text { constant }\end{array}$ \\
\hline 3 & $\mathrm{~T} 2$ & 0.02 & $\mathrm{~s}$ & Differential link lag time constant \\
\hline 4 & $\mathrm{~T} 3$ & 2 & $\mathrm{~s}$ & Integral link lead time constant \\
\hline 5 & $\mathrm{~T} 4$ & 30 & $\mathrm{~s}$ & Integral link lag time constant \\
\hline
\end{tabular}

Simulate generator no-load 5\% step response with parameters in the table above. Then we will study the influence of key parameter of excitation regulator on the dynamic characteristics of generators no-load 5\% step response.

\section{Influence of key parameter of excitation regulator on the dynamic characteristics of generators no-load $5 \%$ step response}

\section{Study on the influence of gain $\mathrm{K}$.}

Keeping other parameters unchanged(T1 $=0.3 、 \mathrm{~T} 2=0.02 、 \mathrm{~T} 3=2 、 \mathrm{~T} 4=30$ ), adjust the value of $\mathrm{K}$ alone, and the change is shown in figure 4. 


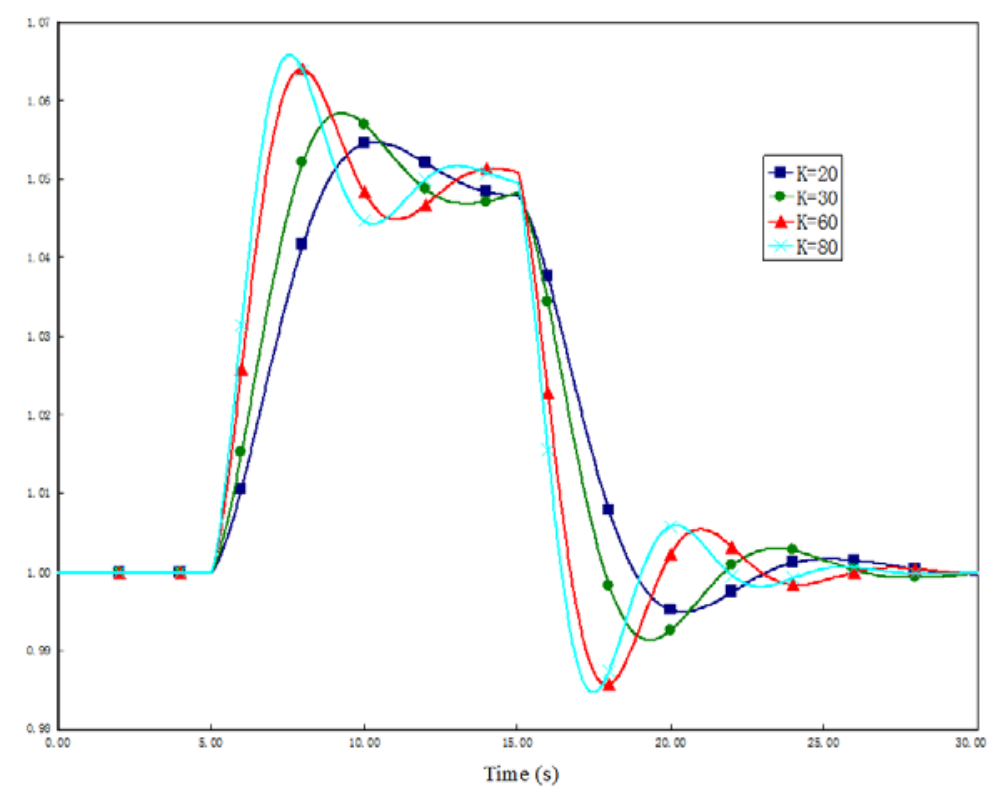

Fig.4 Adjust the value of $\mathrm{K}$ alone

It can be seen that when the other parameters keep unchanged, the larger the $\mathrm{K}$, the smaller the rising time and the peak time, and also the greater the overshoot.

Study on the influence of differential link lead time constant.

Keeping other parameters unchanged $(\mathrm{K}=20 、 \mathrm{~T} 2=0.02 、 \mathrm{~T} 3=2 、 \mathrm{~T} 4=30)$, adjust the value of $\mathrm{T} 1$ alone, and the change is shown in figure 5.

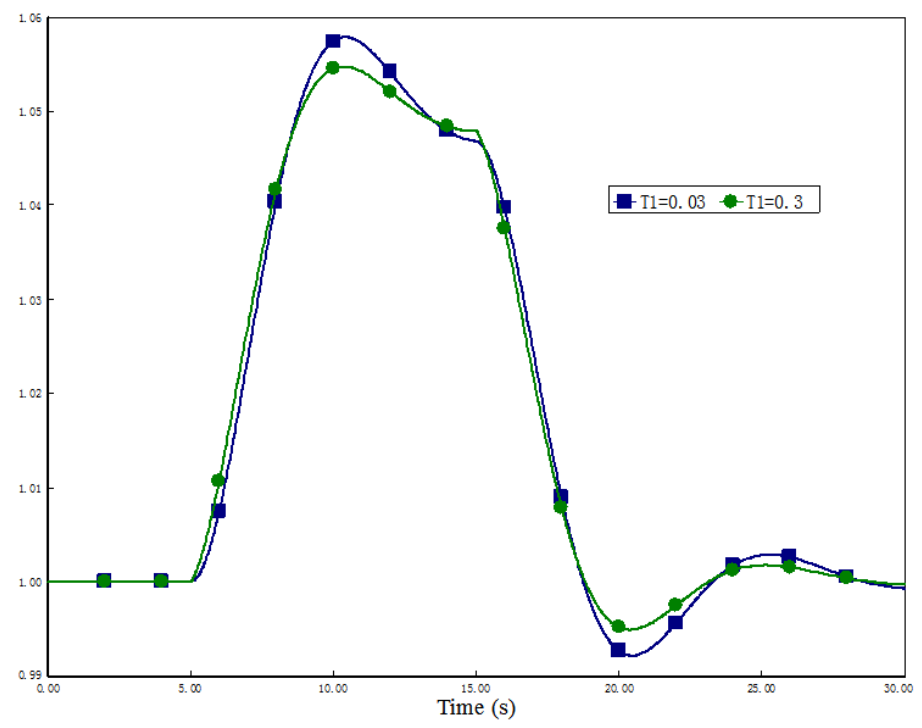

Fig.5 Adjust the value of T1 alone

It can be seen that when the other parameters keep unchanged, the larger the $\mathrm{T} 1$, the smaller the overshoot, and rising time and peak time change little.

Study on the influence of differential link lag time constant.

Keeping other parameters unchanged $(\mathrm{K}=20 、 \mathrm{~T} 1=0.3 、 \mathrm{~T} 3=2 、 \mathrm{~T} 4=30)$, adjust the value of $\mathrm{T} 2$ alone, and the change is shown in figure 6: 


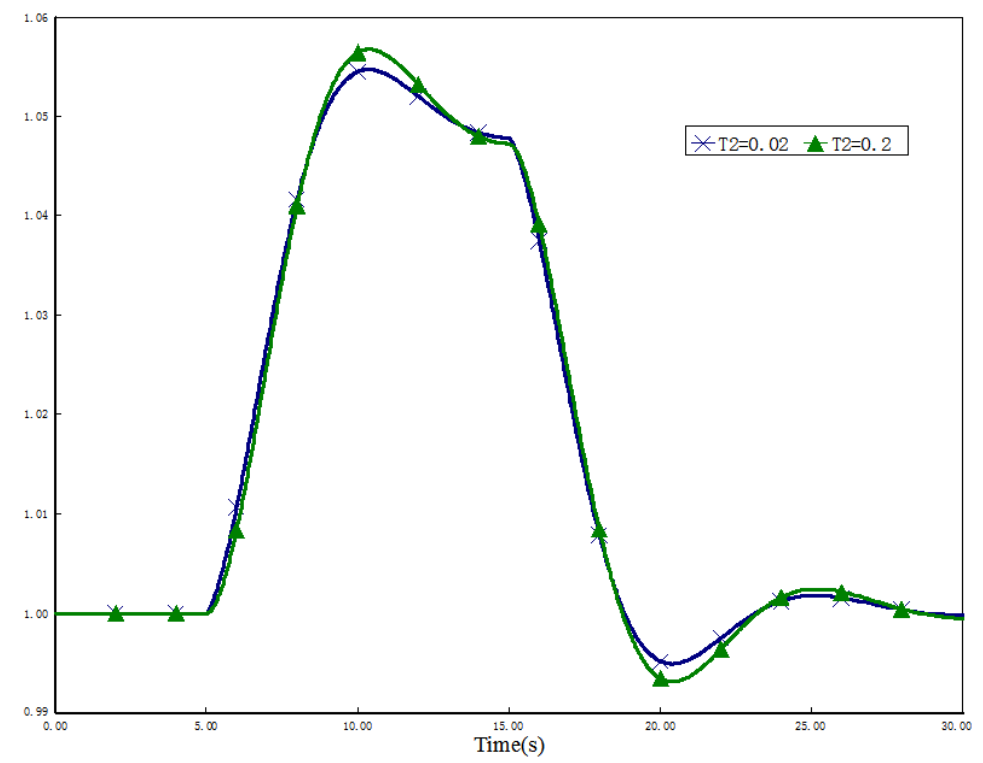

Fig. 6 Adjust the value of T2 alone

It can be seen that when the other parameters keep unchanged, the smaller the T2, the smaller the overshoot, and rising time and peak time change little.

Study on the influence of integral link lead time constant.

Keeping other parameters unchanged $(\mathrm{K}=20 、 \mathrm{~T} 1=0.3 、 \mathrm{~T} 2=0.02 、 \mathrm{~T} 4=30$ ), adjust the value of $\mathrm{T} 3$ alone, and the change is shown in figure 7 :

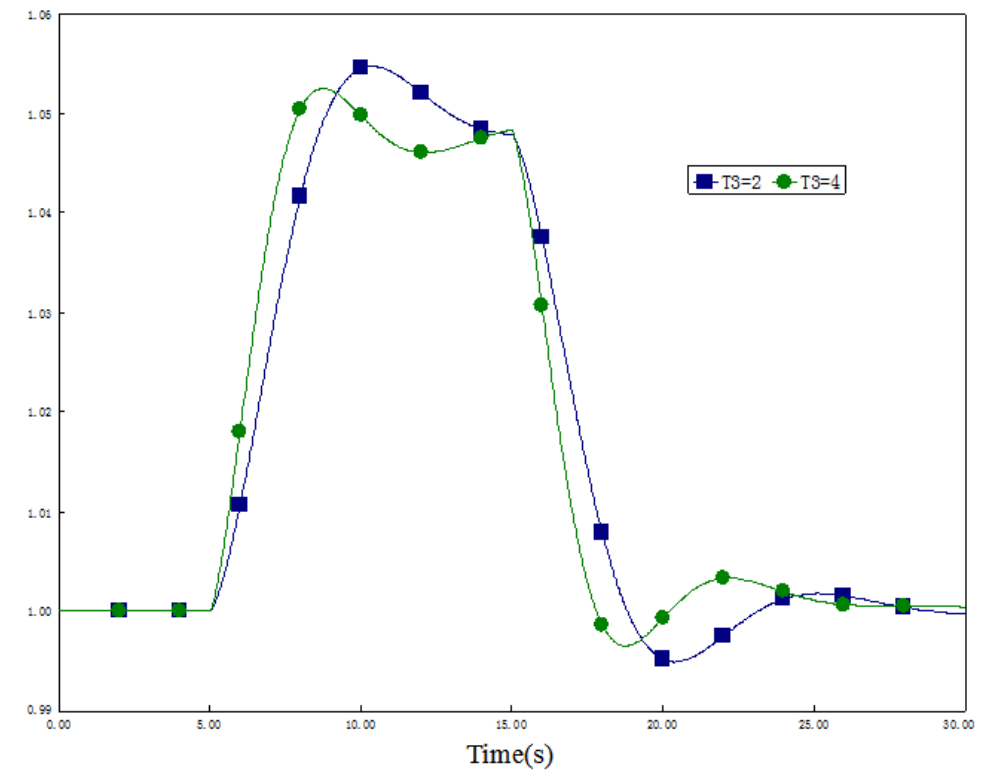

Fig.7 Adjust the value of T3 alone

It can be seen that when the other parameters keep unchanged, the larger the T3, the smaller the overshoot, the rising time and the peak time.

Study on the influence of integral link lag time constant.

Keeping other parameters unchanged $(K=20 、 \mathrm{~T} 1=0.3 、 \mathrm{~T} 2=0.02 、 \mathrm{~T} 3=2)$, adjust the value of $\mathrm{T} 4$ alone, and the change is shown in figure 8: 


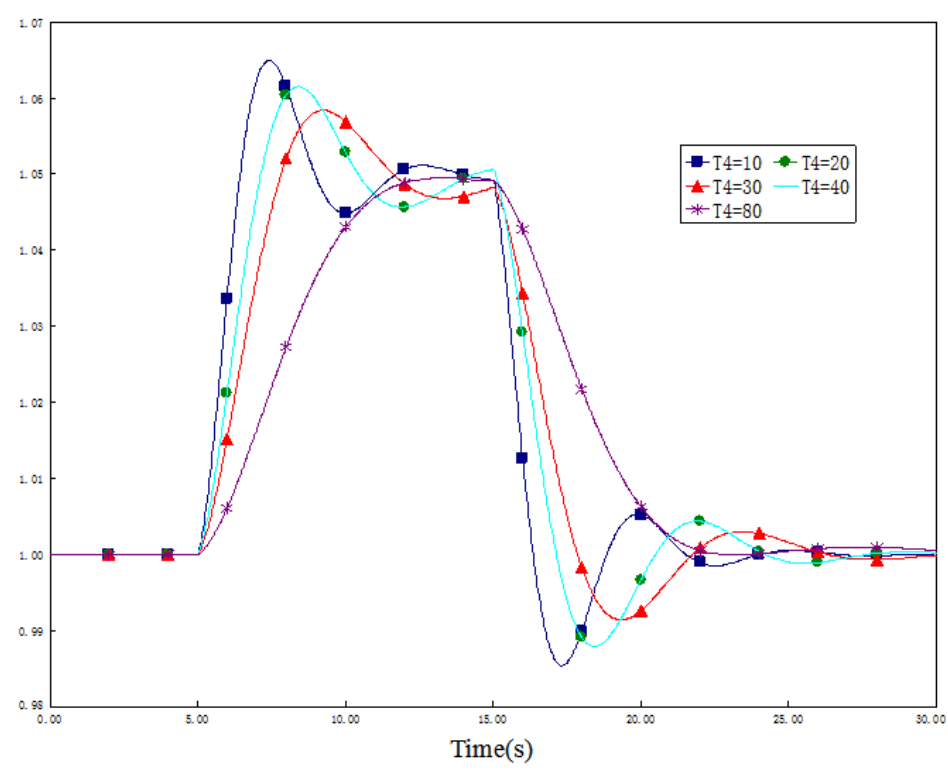

Fig.8 Adjust the value of T4 alone

It can be seen that when the other parameters keep unchanged, the larger the $\mathrm{T} 4$, the smaller the overshoot, and the greater the rising time and the peak time.

\section{Summary and Discussion}

On the basis of simulation and experiments data, we will theoretically discuss the influence of excitation system key parameters on generator transient characteristics.

From the experimental results, without considering the $K_{v}$, the overshoot is positively related to $\mathrm{K}$, and rise time, peak time are inverse related to $\mathrm{K}$; overshoot is inverse related to $\mathrm{T} 1, \mathrm{~T} 2$, $\mathrm{T} 3$, and is positively related to T2; Rising time and peak time are inverse related to T3, and are positively related toT4, but are not related to T1,T2 obviously.

According to control theory, PID correction link in type 3 excitation system transfer function can be simplified as:

$$
\frac{K\left(1+S T_{1}\right)\left(1+S T_{3}\right)}{\left(K_{v}+S T_{2}\right)\left(1+S T_{4}\right)}
$$

Wherein, $\mathrm{T} 1>\mathrm{T} 2, \mathrm{~T} 3<\mathrm{T} 4$, The overall transfer function belongs to a proportional integral derivative controller (PID controller). PID controller has both advantages of PD and PI control, which can achieve no steady-state error, and ensure rapid action. If $\mathrm{K} 、 \mathrm{~T} 1 、 \mathrm{~T} 2 、 \mathrm{~T} 3$ and T4 these parameters fit well, the conditioning effect will be better.

\section{Conclusion}

Based on the above discussion, analysis and experimental results, we can draw the following conclusions:

(1)The overshoot is positively related to $\mathrm{K}$, rise time, peak time are inverse related to $\mathrm{K}$.

(2) Overshoot is inverse related to T1, T2, T3, and is positively related to T2; Rising time and peak time are inverse related to $\mathrm{T} 3$, and are positively related toT4, but are not related to T1,T2 obviously.

All above it's an attempt to adjust one single parameter, however the situation that a plurality of parameters change simultaneously will be more complex, and even differ with the above law, still to be studied. In summary, the influence of excitation system key parameters on generator transient characteristics can be used for identification, checking, and optimization of the generator excitation system parameters. 


\section{Reference}

[1] Wang Liming. Generator Excitation Parameter Measurement and PSS Test Research [J]. Gansu electric power technology, 2006, (4):36-43.

[2] Jiang Liang, Zhu Xiangrong, Yang Liming. Important Function of Excitation Control System in Improving Electric Power System Stability [J]. Machinery manufacturing and automation, 2006, 35(6):163-164.

[3] Guide for Modeling Generator Excitation System. DL/T 1167-2012 : 4-10[S].

[4] You Guangzeng, Si Dajun. Parameter Option for Generator Excitation System in Test and Simulation [J]. Yunnan electric power technology, 2012, 40(6):37-40.

\section{Author Introduce :}

Chen Qiao (1988-), Male, Wuhan, Hubei, Engineer, Master's degree, Main research direction: Electrical Engineering.

E-mail: cq198812@163.com

Cellphone number: 13476169621

QQ: 729912731 